\title{
Application of scattering theory to $P$-wave amplitude fluctuations in the crust
}

\author{
Kazuo Yoshimoto ${ }^{1^{*}}$, Shunsuke Takemura ${ }^{2}$ and Manabu Kobayashi ${ }^{1}$
}

\begin{abstract}
The amplitudes of high-frequency seismic waves generated by local and/or regional earthquakes vary from site to site, even at similar hypocentral distances. It had been suggested that, in addition to local site effects (e.g., variable attenuation and amplification in surficial layers), complex wave propagation in inhomogeneous crustal media is responsible for this observation. To quantitatively investigate this effect, we performed observational, theoretical, and numerical studies on the characteristics of seismic amplitude fluctuations in inhomogeneous crust. Our observations of $P$-wave amplitude for small to moderately sized crustal earthquakes revealed that fluctuations in $P$-wave amplitude increase with increasing frequency and hypocentral distance, with large fluctuations showing up to ten-times difference between the largest and the smallest $P$-wave amplitudes. Based on our theoretical investigation, we developed an equation to evaluate the amplitude fluctuations of time-harmonic waves that radiated isotropically from a point source and propagated spherically in acoustic von Kármán-type random media. Our equation predicted relationships between amplitude fluctuations and observational parameters (e.g., wave frequency and hypocentral distance). Our numerical investigation, which was based on the finite difference method, enabled us to investigate the characteristics of wave propagation in both acoustic and elastic random inhomogeneous media using a variety of source time functions. The numerical simulations indicate that amplitude fluctuation characteristics differ a little between medium types (i.e., acoustic or elastic) or source time function durations. These results confirm the applicability of our analytical equation to practical seismic data analysis.
\end{abstract}

Keywords: Amplitude fluctuation, Ground motion prediction, Crustal inhomogeneity, Seismic scattering, Seismic wave propagation

\section{Introduction}

To predict the strong ground motion of future destructive earthquakes, empirical ground motion prediction equations have been developed and applied to many regions of the world (e.g., $\mathrm{Si}$ and Midorikawa 1999; Douglas 2003). These empirical equations are useful for estimating the average characteristics of seismic amplitude variation with hypocentral distance; however, the observed ground motions (e.g., peak ground velocity) show large scatter around the predictions (e.g., Strasser et al. 2009). It has been suggested that path effects (e.g., Atkinson 2006; Anderson and Uchiyama 2011) are one of the key factors causing this data scatter; source effects (e.g., Brillinger and Preisler 1984; Ripperger et al. 2008)

\footnotetext{
* Correspondence: k_yoshi@yokohama-cu.ac.jp

${ }^{1}$ Department of Material System Science, Graduate School of

Nanobioscience, Yokohama City University, 22-2, Seto, Kanazawa-ku,

Yokohama 236-0027, Japan

Full list of author information is available at the end of the article
}

and site effects (e.g., Chen and Tsai 2002; Lin et al. 2011; Yoshimoto and Takemura 2014) also play a part. Since path effects are governed by lithospheric inhomogeneities, they can be quantitatively estimated using appropriate physical models of wave propagation in inhomogeneous media. However, few studies have tackled this issue (e.g., Nikolaev 1975), mainly because of the difficulties in acquiring high-quality observational data.

In order to investigate the stochastic properties of the Earth's internal inhomogeneities, short-wavelength random inhomogeneities are commonly characterized using stochastic medium models introducing characteristic correlation distances and mean square fractional fluctuations (e.g., Sato et al. 2012). At frequencies below $\sim 1 \mathrm{~Hz}$, spatiotemporal variations in teleseismic $P$-waves, which are considered as plane incident waves into seismic observation arrays, have been analyzed to investigate shortwavelength random inhomogeneities beneath seismic
焦 Springer

(c) 2015 Yoshimoto et al. Open Access This article is distributed under the terms of the Creative Commons Attribution 4.0 International License (http://creativecommons.org/licenses/by/4.0/), which permits unrestricted use, distribution, and reproduction in any medium, provided you give appropriate credit to the original author(s) and the source, provide a link to the Creative Commons license, and indicate if changes were made. 
stations. Flatté and Wu (1988) investigated shortwavelength inhomogeneities in the lithosphere and asthenosphere by analyzing fluctuations in the travel time and amplitudes of teleseismic $P$-waves observed by the Norwegian Seismic Array (NORSAR). Ritter et al. (1998) estimated lithospheric inhomogeneities in the Massif Central region of France, by analyzing frequencydependent intensities of the mean wavefield and the fluctuation wavefield of teleseismic $P$-waves.

A number of theoretical and numerical studies have focused on the amplitudes and phase fluctuations of transmitted waves in random inhomogeneous media (e.g., Rytov et al. 1989; Ishimaru 1997). In these studies, the Rytov approximation has commonly been used to estimate amplitude variance and the phase fluctuations of transmitted waves. Shapiro and Kneib (1993) estimated scattering attenuation in acoustic inhomogeneous media resulting from amplitude fluctuations using the Rytov approximation for the wavefield in a weak fluctuation regime. Hoshiba (2000) demonstrated that the amplitude fluctuations predicted by the Rytov approximation successfully explain the numerical results obtained for scalar plane waves propagating in three-dimensional (3D) Gaussian-type random media. Müller and Shapiro (2003) proposed a formulation to calculate the log-amplitude variance of plane-transmitted waves in anisotropic Gaussian-type random media. However, despite these studies, analyzing the seismograms of local and/or regional earthquakes requires the development of a more realistic physical model that can adequately model spherical wave propagation in realistic random inhomogeneous structures (e.g., von Kármántype random media).

In this study, we first analyzed the seismograms of local earthquakes observed by a dense seismic network in order to reveal the characteristics of $P$-wave amplitude fluctuations in the crust. We then derived an analytical equation for calculating the amplitude fluctuation of spherical waves radiating from a point source in acoustic von Kármán-type random media. The results obtained by this equation were checked using numerical results from finite-difference method (FDM) simulations of seismic wave propagation. Finally, we considered the extent to which observed $P$-waves amplitude fluctuations in the crust were explained by the predictions of our analytical equation. The results confirmed the applicability of our analytical equation to practical seismic data analysis.

\section{Seismic observations}

Data

To investigate the characteristics of $P$-wave amplitude fluctuations for crustal earthquakes, we analyzed threecomponent velocity seismograms recorded at $\mathrm{Hi}$-net borehole stations in the Chugoku region of southwestern Japan (Fig. 1), following the methodology of Kobayashi et al. (2015). Hi-net is a high-sensitivity seismogram network operated by the National Research Institute for Earth Science and Disaster Prevention, Japan (Okada et al. 2004). By adding three earthquakes to the Kobayashi et al. (2015)'s dataset, we selected 13 small to moderately sized strike-slip earthquakes (Table 1), for which mechanisms were consistently reported by centroid moment tensor (CMT) solutions of F-net (Fukuyama et al. 1998) and first motion focal mechanisms by the Japan Meteorological Agency.

\section{$P$-wave amplitude fluctuations}

We measured maximum $P$-wave amplitudes from the three-component vector amplitude of the filtered velocity seismograms. Following the method of Kobayashi et al. (2015), in order to eliminate the effects of different earthquake source sizes and site amplifications, the observed maximum $P$-wave amplitudes were normalized by the averaged $S$-wave coda amplitude at lapse times of 60-70 s, which were based on the coda normalization method (e.g., Yoshimoto et al. 1993). Hereafter, we refer to the coda-normalized maximum $P$-wave amplitude as " $P$-wave amplitude".

Characteristics of $P$-wave amplitudes were investigated as a function of hypocentral distance in the frequency bands of $1-2$ and $2-4 \mathrm{~Hz}$ (Fig. 2a). The observed $P$-wave amplitudes decreased with increasing hypocentral distance owing to the effects of geometrical spreading, intrinsic attenuation, and scattering attenuation. As observed by Kobayashi et al. (2015), our results showed large data scatter, reflecting a non-isotropic source radiation pattern. Data corresponding to large $\left|F_{P}\right|$ (the magnitude of $P$-wave radiation pattern coefficient expected from CMT solutions in the homogeneous medium; Aki and Richards 2002) were distributed in higher positions and vice versa.

By selecting only data with similar and large $P$-wave radiation pattern coefficients $\left(\left|F_{P}\right| \geq 0.7\right)$, the increase in data scatter with increasing hypocentral distance, along with the frequency changes, became clearer (Fig. 2b). Using an observational property of the small data scatter at short hypocentral distances (except for one outlier at $\sim 15 \mathrm{~km}$ in the $1-2-\mathrm{Hz}$ band), which is likely to indicate well-calibrated source magnitudes, we were able to investigate the propagation dependence of $P$-wave amplitude fluctuations in the inhomogeneous crust.

\section{Analytical methods}

\section{Analytical equation for amplitude fluctuations}

Following the mathematical framework of Ishimaru (1997, Chapters 16-18, pp. 321-379), we derived an analytical equation to evaluate the amplitude fluctuations of spherical waves radiated by a point source in 3D inhomogeneous acoustic media. For simplicity, we assumed an 


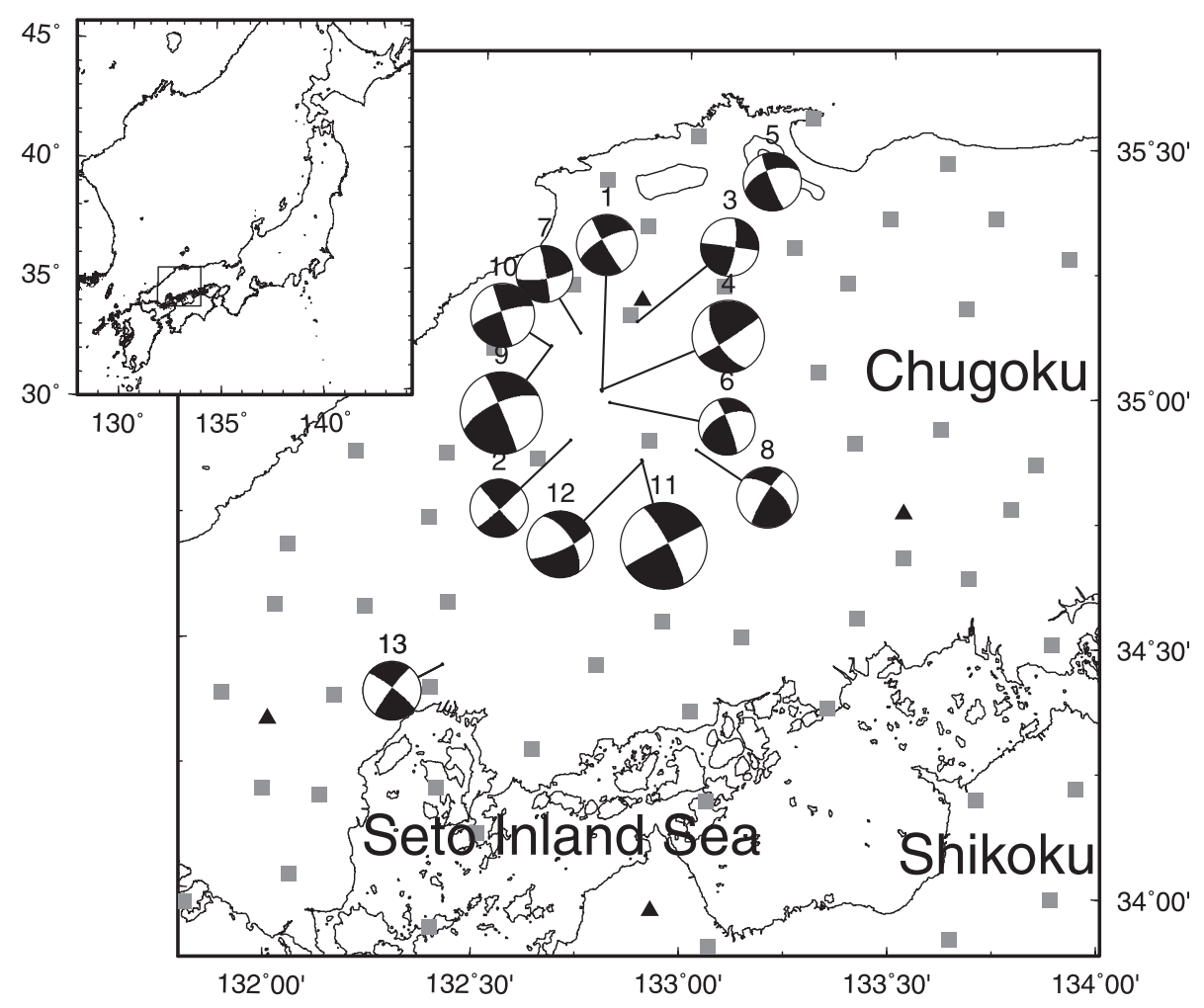

Fig. 1 Distribution of seismic stations and earthquakes. Map showing the distribution of seismic stations and earthquakes in the Chugoku region of southwestern Japan. Grey squares and black triangles denote Hi-net and F-net stations, respectively. The focal mechanisms of the earthquakes represent the centroid moment tensor (CMT) solutions of F-net. The GMT software (Wessel and Smith 1998) was used to draw the map.

infinite inhomogeneous volume, in which velocity $c(\mathbf{r})$ fluctuated as a function of position $\mathbf{r}$. We assumed a weak velocity fluctuation, which was isotropic and uniformly distributed in space:

$$
c(\mathbf{r})=c_{0}(1+\xi(\mathbf{r}))
$$

where $c_{0}$ is the background velocity and $\xi(\mathbf{r})$ is the fractional fluctuation of velocity. The fractional fluctuation should be small or $|\xi(\mathbf{r})| \ll 1$ and $\langle\xi(\mathbf{r})\rangle=0$, where the symbol \langle\rangle denotes the statistical ensemble average.

Under these conditions, wave propagation in 3D inhomogeneous acoustic media can be evaluated using a scalar wave equation:

$$
\Delta u(\mathbf{r}, t)-\frac{1}{c_{0}^{2}}(1-2 \xi(\mathbf{r})) \frac{\partial^{2} u(\mathbf{r}, t)}{\partial t^{2}}=0
$$

where $u(\mathbf{r}, t)$ is the scalar wavefield, $t$ is time, and $\Delta$ is the Laplacian operator. For the solution of this equation, we assumed a time-harmonic spherical wave propagating outward from a point source. We employed a first-order Rytov approximation solution:

$$
u(\mathbf{r})=u_{0}(\mathbf{r}) \exp \varphi(\mathbf{r})
$$

$$
\varphi(\mathbf{r}) \equiv \chi(\mathbf{r})+i \phi(\mathbf{r}) \equiv-2 k^{2} \int_{\mathbf{v}^{\prime}} G\left(\mathbf{r}-\mathbf{r}^{\prime}\right) \xi\left(\mathbf{r}^{\prime}\right) \frac{u_{0}\left(\mathbf{r}^{\prime}\right)}{u_{0}(\mathbf{r})} d \mathbf{V}^{\prime}
$$

The exponential term in Eq. (3) represents the amplitude and phase modulation due to inhomogeneities superposed on the homogeneous background medium. For simplicity, the time-dependent term $\exp (-\mathrm{i} \omega t)$ is not explicitly written: the symbol $i$ is the imaginary unit and $\omega$ is the angular frequency of a time-harmonic spherical wave. In Eq. (4), $k$ is the wavenumber and symbols $u_{0}(\mathbf{r})$ and $G$ are the wavefield and Green's function, respectively, in the homogeneous background medium. The Green's function is given by:

$$
G\left(\mathbf{r}-\mathbf{r}^{\prime}\right)=\frac{\exp \left(i k\left|\mathbf{r}-\mathbf{r}^{\prime}\right|\right)}{4 \pi\left|\mathbf{r}-\mathbf{r}^{\prime}\right|}
$$

The 3D integral of Eq. (4) becomes non-zero only for the volume element $d \mathbf{V}^{\prime}$, for which a fractional fluctuation of velocity exists. Equation (4) is identical to equation (17-23) of Ishimaru (1997), except for the negative sign arising from the different definitions of the fractional fluctuation (Eq. (1)) and the fluctuation of the refractive index of Ishimaru (1997). 
Table 1 Earthquake catalog

\begin{tabular}{|c|c|c|c|c|c|c|c|c|}
\hline No. & Date/time(JST) & Lat. $\left({ }^{\circ} \mathrm{N}\right)$ & $\log \left({ }^{\circ} \mathrm{E}\right)$ & Depth(km) & Strike $\left({ }^{\circ}\right)$ & $\operatorname{Dip}\left(^{\circ}\right)$ & $\operatorname{Rake}\left(^{\circ}\right)$ & $M_{W}$ \\
\hline 1 & $\begin{array}{l}\text { 2005/03/19, } \\
15: 03: 46.92\end{array}$ & 132.80 & 35.01 & 5 & 149 & 86 & -20 & 3.6 \\
\hline 2 & $\begin{array}{l}\text { 2005/07/28, } \\
09: 11: 11.30\end{array}$ & 132.72 & 34.91 & 8 & 138 & 88 & 3 & 3.5 \\
\hline 3 & $\begin{array}{l}\text { 2006/11/26, } \\
12: 58: 35.01\end{array}$ & 132.88 & 35.15 & 11 & 277 & 89 & 165 & 3.5 \\
\hline 4 & $\begin{array}{l}\text { 2007/05/13, } \\
08: 13: 54.79\end{array}$ & 132.79 & 35.01 & 8 & 56 & 86 & 152 & 4.3 \\
\hline 5 & $\begin{array}{l}\text { 2007/10/14, } \\
23: 38: 50.08\end{array}$ & 133.20 & 35.43 & 5 & 157 & 85 & -35 & 3.5 \\
\hline 6 & $\begin{array}{l}\text { 2010/01/21, } \\
08: 50: 36.79\end{array}$ & 132.81 & 34.99 & 5 & 338 & 83 & 36 & 3.4 \\
\hline 7 & $\begin{array}{l}\text { 2010/06/07, } \\
\text { 07:43:01.09 }\end{array}$ & 132.74 & 35.12 & 8 & 349 & 87 & -14 & 3.4 \\
\hline 8 & $\begin{array}{l}\text { 2011/02/07, } \\
00: 36: 15.77\end{array}$ & 133.03 & 34.89 & 5 & 211 & 81 & 149 & 3.6 \\
\hline 9 & $\begin{array}{l}\text { 2011/06/04, } \\
01: 57: 31.01\end{array}$ & 132.67 & 35.10 & 11 & 336 & 87 & 22 & 4.9 \\
\hline 10 & $\begin{array}{l}\text { 2011/06/16, } \\
\text { 23:32:21.15 }\end{array}$ & 132.67 & 35.10 & 8 & 161 & 89 & -12 & 3.8 \\
\hline 11 & $\begin{array}{l}\text { 2011/11/21, } \\
19: 16: 29.59\end{array}$ & 132.89 & 34.87 & 11 & 241 & 89 & 170 & 5.2 \\
\hline 12 & $\begin{array}{l}\text { 2011/11/25, } \\
04: 52: 24.33\end{array}$ & 132.90 & 34.87 & 5 & 64 & 76 & -150 & 4.0 \\
\hline 13 & $\begin{array}{l}\text { 2013/01/08, } \\
\text { 20:19:32.76 }\end{array}$ & 132.42 & 34.46 & 20 & 216 & 84 & 172 & 3.5 \\
\hline
\end{tabular}

JST Japan Standard Time

In Eq. (4), $\chi(\mathbf{r})$ and $\phi(\mathbf{r})$ are assumed to be real functions. The function $X(\mathbf{r})$ is related to the fluctuation in amplitude as follows:

$$
\chi(\mathbf{r})=\ln \left|\frac{u(\mathbf{r})}{u_{0}(\mathbf{r})}\right|
$$

It should be mentioned that, in case of $|X(\mathbf{r})| \ll$ $1,|\chi(\mathbf{r})|$ is approximately equal to $\left|u(\mathbf{r})-u_{0}(\mathbf{r})\right| /\left|u_{0}(\mathbf{r})\right|$. We here defined the variance of the amplitude level (hereafter, amplitude level variance) as:

$$
\sigma_{x}^{2} \equiv\left\langle(x-\langle x\rangle)^{2}\right\rangle
$$

This quantity is one-fourth of the scintillation index (Ishimaru 1997), which represents the variance of wave intensity. Shapiro and Kneib (1993) derived a simple relationship between the amplitude spectra of $u(\mathbf{r}), U$, and the amplitude level variance:

$$
\sigma_{X}^{2}=2(\ln \langle U\rangle-\langle 1 \mathrm{n} U\rangle)
$$

This relationship is useful for estimating $\sigma_{X}^{2}$ when we do not have rigorous information on $u_{0}(\mathbf{r})$.

Assuming a line-of-sight propagation of a spherical wave (Ishimaru 1997), we specifically considered the amplitude level variance at distance $L$ from the point source:

$$
\sigma_{X}^{2}=\left\langle(X-\langle x\rangle)^{2}\right\rangle=4 \pi^{2} k^{2} \int_{0}^{L} d \eta \int_{0}^{\infty} v \sin ^{2}\left[\frac{\gamma(L-\eta)}{2 k} v^{2}\right] \Phi_{\xi}(v) d v
$$

where $\gamma \equiv \eta / L$, and $\Phi_{\xi}$ is the power spectral density function of $\xi$. Equation (9) is same as equation (18-5) of Ishimaru (1997). Assuming a von Kármán-type random media (e.g., Sato et al. 2012) for characterizing the stochastic properties of $\xi, \Phi_{\xi}$ can be expressed by a function of wavenumber $v$ as follows:

$$
\Phi_{\xi}(v)=\alpha \varepsilon^{2}\left(v^{2}+a^{-2}\right)^{-\beta}
$$

where $\varepsilon^{2} \equiv\left\langle\xi(\mathbf{r})^{2}\right\rangle, a$ is the correlation distance, and parameters $\alpha$ and $\beta(3 / 2 \sim 5 / 2)$ are positive constants. The value of $\alpha$ may be set in relation to $\beta$ as follows:

$$
\alpha=\pi^{-\frac{3}{2}} a^{-2\left(\beta-\frac{3}{2}\right)} \Gamma(\beta) \Gamma\left(\beta-\frac{3}{2}\right)^{-1}
$$

where $\Gamma()$ is the gamma function. It should be noted that this definition of the power spectral density function, used 

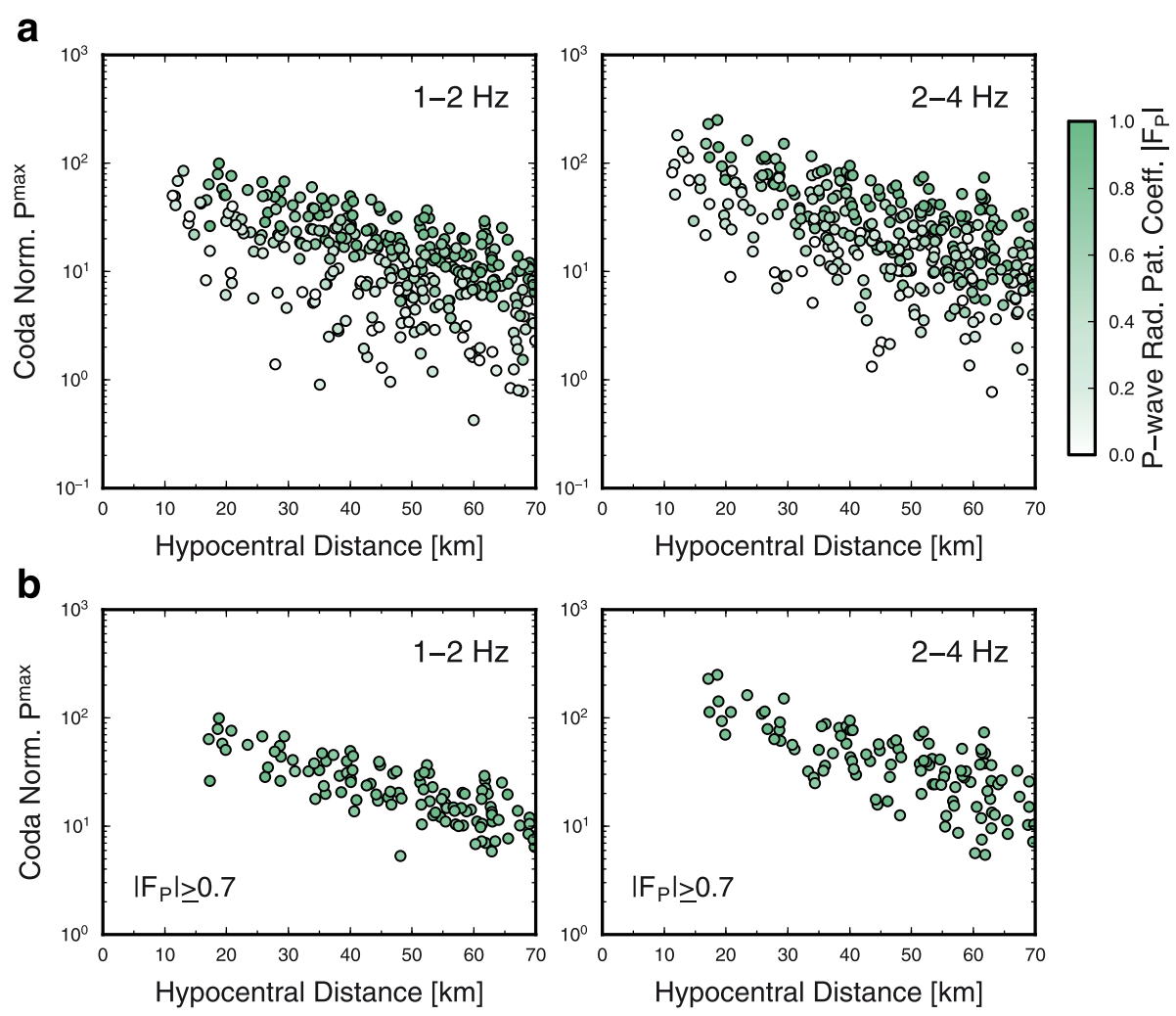

Fig. 2 Distance change in coda-normalized $P$-wave amplitudes. a Coda-normalized $P$-wave amplitudes in the 1-2-and 2-4-Hz bands, as measured from crustal earthquakes (Fig. 1). Values of $\left|F_{P}\right|$ are the magnitudes of the $P$-wave radiation pattern coefficients expected from the centroid moment tensor (CMT) solutions of the F-net. $\mathbf{b}$ The same as a except with a limitation of $\left|F_{P}\right| \geq 0.7$

by Ishimaru (1997), differs in $(2 \pi)^{-3}$ with that of Sato et al. (2012). Substitution of equation (10) into (9) and integration over $v$ gives the amplitude level variance at distance $L$ as a simple integral form:

$$
\sigma_{X}^{2}=\alpha \pi^{2} k^{2} \varepsilon^{2} a^{2(\beta-1)} \int_{0}^{L} d \eta\left[\frac{1}{\beta-1}-\operatorname{Re} \psi\left(1,2-\beta, i \frac{\gamma(L-\eta)}{k a^{2}}\right)\right]
$$

where $\operatorname{Re} \psi()$ is the real part of the confluent hypergeometric function of the second kind (Abramowitz and Stegun 1964, equation 13.2 .5 , p. 505). If we assume exponential-type random media $\left(\alpha=\pi^{-2} a^{-1}\right.$ and $\beta=2$; e.g., Sato et al. 2012), Eq. (12) can be simplified to:

$$
{ }^{\operatorname{Exp}} \sigma_{\mathrm{X}}^{2}=k^{2} \varepsilon^{2} a\left[L-\int_{0}^{L} d \eta \operatorname{Re} \psi\left(1,0, i \frac{\gamma(L-\eta)}{k a^{2}}\right)\right]
$$

From the same geometric consideration of wave propagation as in Ishimaru (1997), Eqs. (12) and (13) can be used for a plane wave case when $\gamma=1$.

\section{Evaluation of amplitude fluctuations}

Power spectral density of von Kármán-type random media varies with parameter $\beta$ (Fig. 3a). Reflecting this property, the amplitude level variance increases with increasing $\beta$ value (Fig. 3b). For the comparative analysis using numerical and observational data in the following sections, we assumed stochastic parameters of $a=1 \mathrm{~km}$ and $\varepsilon=0.03$ (Kobayashi et al. 2015) for crustal inhomogeneities. The hypocentral distance dependence of amplitude level variance arises from the increase in the power spectral density components of inhomogeneity at small wavenumbers, which causes the phase modulation of a propagating wave (Uscinski 1977). The dotted line indicates the saturation level of $\sigma_{X}^{2}$, which is equal to 0.17 , in the strong wavefield fluctuation regime, where the log-amplitude variance tends to the constant, or scintillation index saturates (e.g., Müller and Shapiro 2003). Below this saturation level, estimations from the analytical equation derived in this study may be useful for data analysis.

To better understand the $k$ dependence of $\sigma_{x}^{2}$ for exponential-type random media, in relation with the contribution of the integral part of Eq. (13), different $k$ values of $\pi / 4, \pi / 2, \pi, 2 \pi$, and $4 \pi \mathrm{km}^{-1}$ (corresponding to 


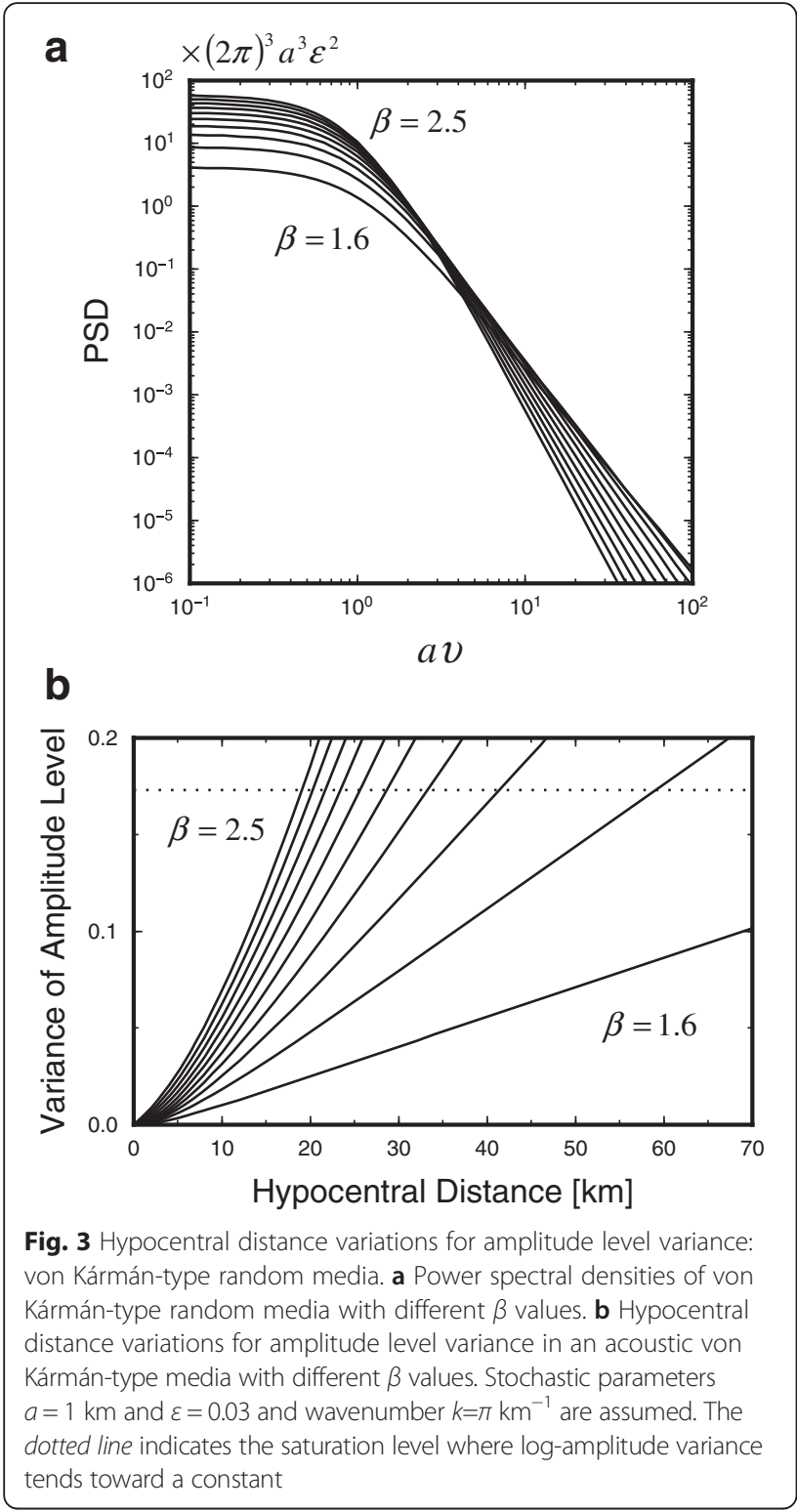

frequencies of $3 / 4,3 / 2,3,6$, and $12 \mathrm{~Hz}$, respectively, for the inhomogeneous medium with a background velocity of $6.0 \mathrm{~km} / \mathrm{s}$ ) are considered (Fig. 4). The results show that the amplitude level variance increases for waves with large wavenumbers or short wavelengths. For example, in the case of $k=\pi$, a spherical wave propagating outward from a point source reaches the strong wavefield fluctuation regime at a hypocentral distance of $\sim 30 \mathrm{~km}$, indicating that the deterministic analysis of wave amplitude is no longer applicable.

\section{FDM simulations}

To verify the applicability of our analytical equation for amplitude fluctuations to observed seismic data, we conducted a set of numerical simulations of seismic wave

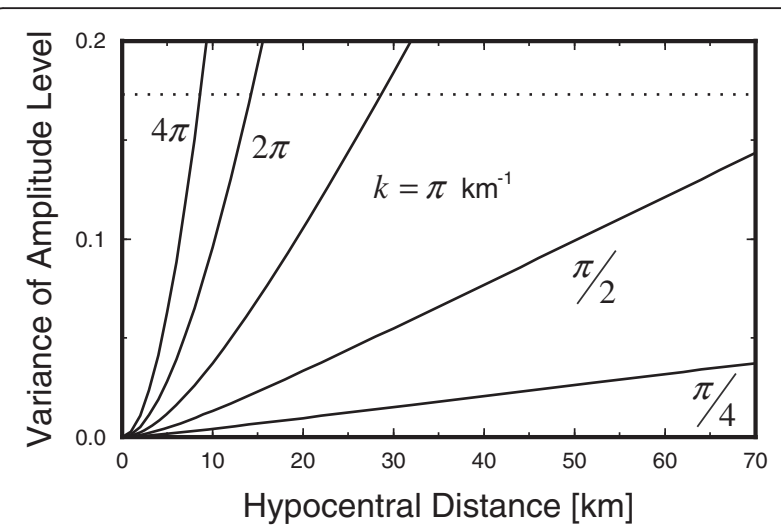

Fig. 4 Hypocentral distance variations for amplitude level variance: exponential-type random media. Hypocentral distance variations for amplitude level variance in acoustic exponential-type random media with wavenumber values of $\pi / 4, \pi / 2, \pi, 2 \pi$, and $4 \pi \mathrm{km}^{-1}$. Stochastic parameters $a=1 \mathrm{~km}$ and $\varepsilon=0.03$ are assumed. The dotted line indicates the saturation level where log-amplitude variance tends toward a constant

propagation in 3D random velocity inhomogeneous media using the FDM technique. We adopted a fourthorder staggered-grid parallel FDM code (Takemura et al. 2015) and simulation models that were similar to those used in Kobayashi et al. (2015). We investigated different types of inhomogeneous media (Table 2). The model volume of $200 \times 200 \times 120 \mathrm{~km}^{3}$, which was common to all medium models, was discretized using a uniform grid interval of $0.1 \mathrm{~km}$. To conduct 3D simulations effectively, we employed a parallel FDM simulation code based on a domain partitioning procedure using large number of processors and the Message Passing Interface (Furumura and Chen 2004). All simulations were conducted on the computer system of the Earthquake and Volcano Information Centre at the Earthquake Research Institute, The University of Tokyo.

We assumed an explosive-type point source, which radiated $P$-waves isotropically. Source time functions (Table 3; Fig. 5) were represented by sin and Ricker wavelets (e.g., Mavroeidis and Papageorgiou 2003). Seismic sources were located at the center of the model.

Table 2 Types of inhomogeneous media

\begin{tabular}{|c|c|c|c|c|c|c|c|}
\hline \multirow[t]{2}{*}{ No. } & \multirow{2}{*}{$\begin{array}{l}\text { Medium- } \\
\text { type }\end{array}$} & \multicolumn{2}{|c|}{ Randomness } & \multicolumn{3}{|c|}{ Background parameters } & \multirow{2}{*}{$\begin{array}{l}\text { Variable } \\
\text { parameters }\end{array}$} \\
\hline & & $a(\mathrm{~km})$ & $\varepsilon$ & $\begin{array}{l}P \text {-wave } \\
\text { velocity } \\
(\mathrm{km} / \mathrm{s})\end{array}$ & $\begin{array}{l}\text { S-wave } \\
\text { velocity } \\
(\mathrm{km} / \mathrm{s})\end{array}$ & $\begin{array}{l}\text { Density } \\
\left(\mathrm{g} / \mathrm{cm}^{3}\right)\end{array}$ & \\
\hline A & Acoustic & 1.0 & 0.03 & 6.0 & 0 & 2.6 & $\begin{array}{l}P \text {-wave } \\
\text { velocity }\end{array}$ \\
\hline \multirow[t]{2}{*}{ B } & Elastic & 1.0 & 0.03 & 6.0 & 3.5 & 2.6 & $\begin{array}{l}P \text { - and S-wave } \\
\text { velocity }\end{array}$ \\
\hline & & & & & & & Density \\
\hline C & Elastic & 1.0 & 0.03 & 6.0 & 3.5 & 2.6 & $\begin{array}{l}P \text { - and S-wave } \\
\text { velocity }\end{array}$ \\
\hline
\end{tabular}


Table 3 Source time functions

\begin{tabular}{llcl}
\hline No. & Type & Frequency $(\mathrm{Hz})$ & Duration $(\mathrm{s})$ \\
\hline 1 & Sin & 1.5 & 9 \\
2 & Sin & 3.0 & 1 \\
3 & $\operatorname{Sin}$ & 3.0 & 2 \\
4 & Sin & 3.0 & 3 \\
5 & Sin & 3.0 & 6 \\
6 & Sin & 3.0 & 9 \\
7 & Ricker & 1.5 & $\sim 0.67$ \\
8 & Ricker & 3.0 & $\sim 0.33$ \\
\hline
\end{tabular}

Under these calculation conditions, our simulations examined seismic wave propagation at frequencies below $f$ $=4.375 \mathrm{~Hz}$ (wavelength $\lambda=0.8 \mathrm{~km}$ ) by sampling 8 grid points per minimum $S$-wavelength (e.g., Takemura et al. 2015). To obtain the statistical properties of the highfrequency seismic wavefield, we calculated seismograms at dense virtual seismic stations with a uniform interval of $2.5 \mathrm{~km}$ (6400 stations), which were distributed at the same depth as the seismic source.

To construct realistic inhomogeneous crustal models, we employed stochastic random elastic fluctuations characterized by an exponential-type power spectral density function in the wavenumber domain $(\beta=2$ of the von Kármán-type power spectral density function). We assumed $a=1 \mathrm{~km}$ and $\varepsilon=0.03$, which were estimated for the crust in the Chugoku region by Kobayashi et al. (2015). Constructed stochastic random elastic fluctuations were embedded over mean background elastic parameters. We assumed a linear relationship of fluctuations among the $P$-wave velocity, $S$-wave velocity, and the density from Birch's law (Birch 1961) for medium model B, whereas density fluctuations was not allowed in medium model C. For the construction of the acoustic random inhomogeneous medium, which we used in the numerical verification of the analytical equation, we set the $S$-wave velocity of medium model C to $0 \mathrm{~km} / \mathrm{s}$.

\section{Results and discussion}

\section{Analytical predictions and FDM simulation results}

An example of the spatial distribution of the maximum amplitudes of quasi-time-harmonic waves (no. 1 and no. 6 source time functions) radiated isotropically from a point source in the random inhomogeneous acoustic medium (model A) is shown in Fig. 6a. The amplitude of the spherical wave showed very weak azimuthal variations at the hypocentral region; however, there were distinct azimuthal variations at large hypocentral distances. The region in which relatively large amplitudes were observed appeared as a spoke-like structure radiating from the source, implying that ray-bending in the hypocentral region strongly affected the construction of the nonisotropic azimuthal distribution, or spatial variation, of the spherical wave's amplitude at large hypocentral distances. The spoke-like structure became indistinct at increasing hypocentral distances owing to successive ray-

\section{a}

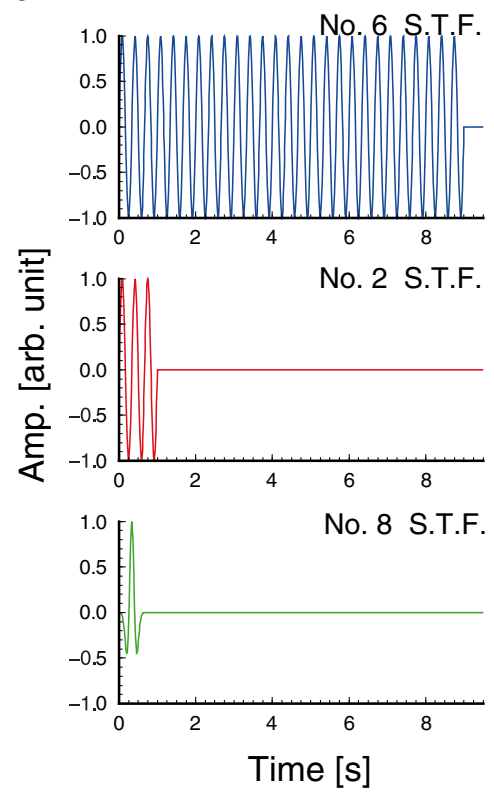

b

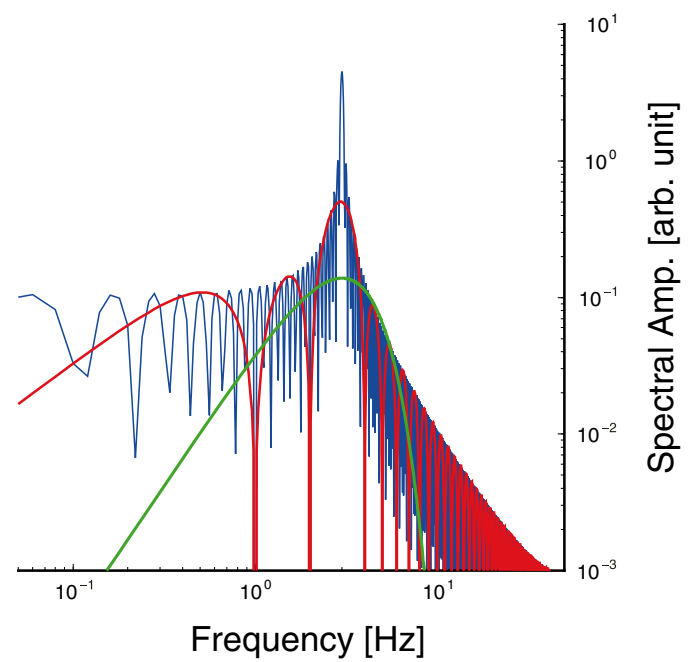

Fig. 5 Waveform examples for different source time functions and their amplitude spectra. a Waveforms for the no. 6, 2, and 8 source time functions (top, middle, and bottom, respectively). b Amplitude spectra for the waveforms of the no. 6, 2, and 8 source time functions. Line spectrum representing periodic signal is well developed for the waveform of the no. 6 source time function 

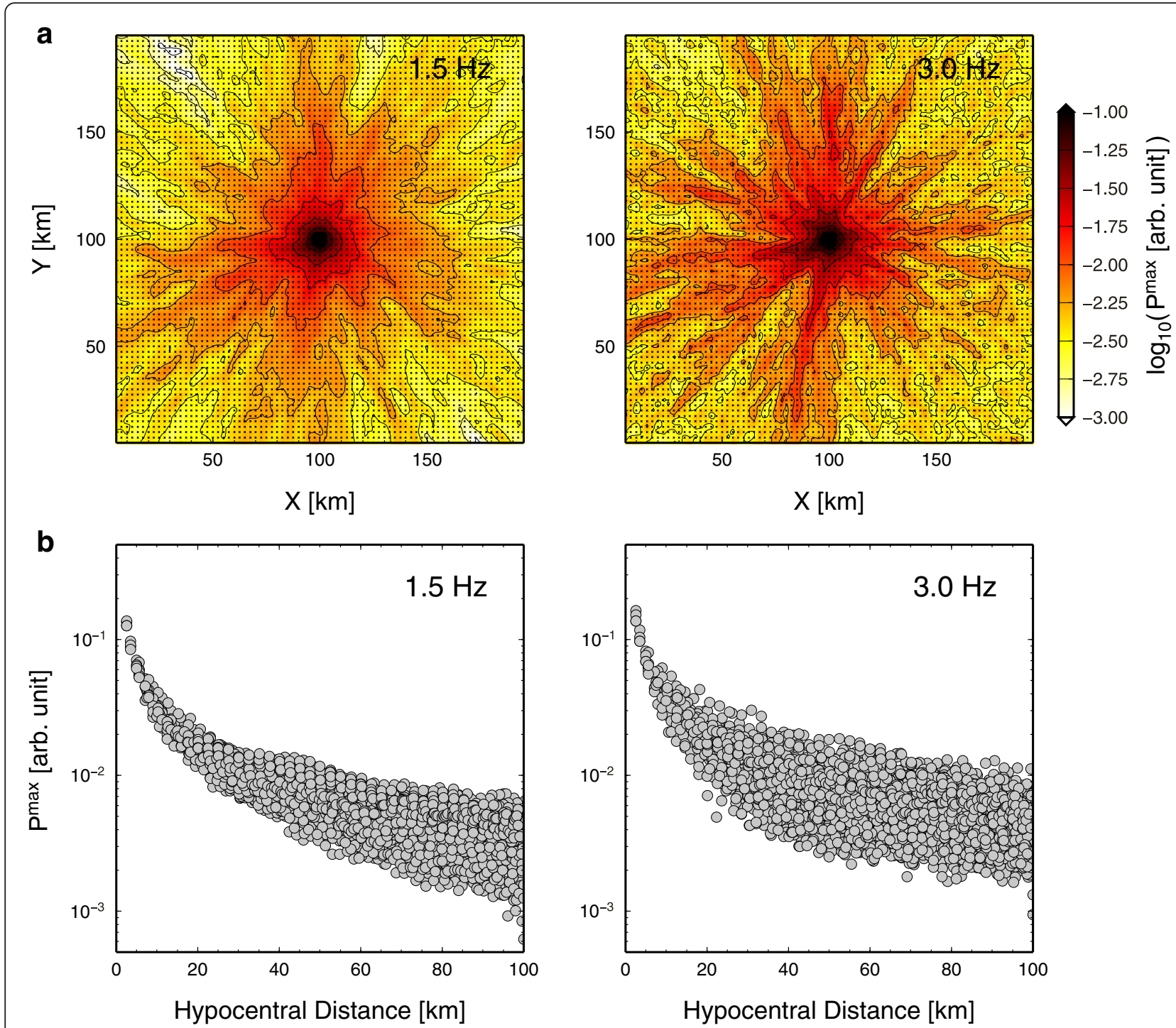

Fig. 6 Spatial and hypocentral distance distribution of wave amplitudes in random inhomogeneous media. Spatial and hypocentral distance distribution of wave amplitudes in random inhomogeneous media using medium model A and the no. 1 and 6 source time functions for FDM simulations. a Spatial distribution of wave amplitudes using sine wavelets for frequencies of 1.5 and $3.0 \mathrm{~Hz}$. b Hypocentral distance variation of wave amplitudes for frequencies of 1.5 and $3.0 \mathrm{~Hz}$. Data are normalized by the wave amplitude at the source region

bending processes during wave propagation. The spatial variations of wave amplitude were stronger for the $3-\mathrm{Hz}$ simulation than for the $1.5-\mathrm{Hz}$ simulation.

The hypocentral distance variations of the maximum amplitudes in the random inhomogeneous acoustic media (model A) are shown in Fig. 6b. To collect sufficient data, we conducted four FDM simulations using four different realizations of random media. The apparent attenuation of maximum amplitudes was due to geometrical spreading and scattering effects. An increase in the variation of amplitude was observed with increasing hypocentral distance; however, the variation in amplitude was barely detectable at small hypocentral distances. As a result, we observed up to ten-times difference between the largest and the smallest $P$ - wave amplitudes. The increasing variation in amplitude with increasing hypocentral distance appeared to saturate at a certain distance $(\sim 30 \mathrm{~km})$ in the $3-\mathrm{Hz}$ simulation.

The distribution of log-amplitudes at each hypocentral distance resembled a normal distribution (Fig. 7). This is similar to the findings of Hoshiba (2000), obtained using phase screen method simulations for plane wave propagation in 3D Gaussian-type random acoustic media. Under the first-order Rytov approximation, the log-amplitude of randomly scattered waves statistically follows a normal distribution (e.g., Andrews and Phillips 2005). Furthermore, our results also showed a broadening of the log-amplitude distribution with increasing frequency. 

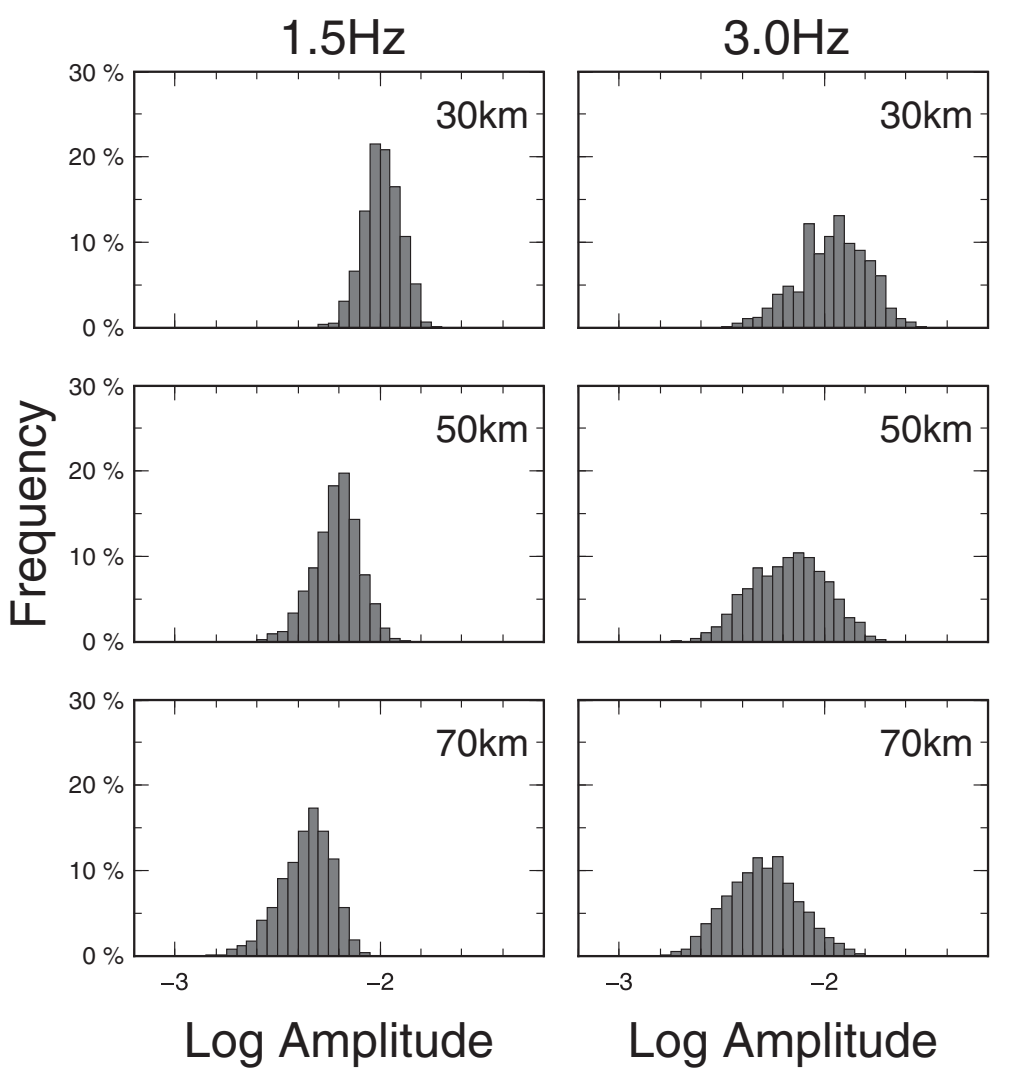

Fig. 7 Distribution of wave amplitudes in random inhomogeneous media. Log-amplitude distributions obtained at distances of $30 \pm 1,50 \pm 1$, and $70 \pm 1 \mathrm{~km}$ by the FDM simulations (Fig. 6b). The log-amplitudes are shown using an arbitrary unit

To quantify the variation in wave amplitude, we used the amplitude level variance $\sigma_{\chi}^{2}$, as defined by Eq. (8). For simplicity and practical convenience, instead of using $U$ in this equation, we used the maximum amplitude for the analysis of time-domain waveforms. The value of $\sigma_{X}^{2}$ increased with hypocentral distance, with the increase rate differing between the 1.5- and 3-Hz waves (Fig. 8). The rates of increase and the absolute values of the amplitude level variance were smaller for $1.5-\mathrm{Hz}$ waves (no. 1 source time function). For this lower-frequency wave, the amplitude level variance monotonically increased over the whole hypocentral distance range. In contrast, for the $3-\mathrm{Hz}$ wave (no. 6 source time function), the amplitude level variance became saturated at a distance of $\sim 30 \mathrm{~km}$, after which we observed a roughly flat data distribution at large hypocentral distances. The results also showed that the analytical evaluations of Eq. (13) can explain the results of the FDM simulations, with the exception of the slight underestimation in the $1.5-\mathrm{Hz}$ wave data. We interpreted that the hypocentral distance range where the amplitude level variance of the $3-\mathrm{Hz}$ wave became saturated corresponded to the strong wavefield fluctuation regime, where the weak fluctuation approximation of Eq. (13) was not appropriate.

\section{Amplitude fluctuations: source wavelet dependence}

Earthquake sources radiate non-time-harmonic waves, especially at low frequencies below the corner frequency. Thus, for the application of Eq. (13) to substantial earthquake data, it was necessary to check the extent to which the time-harmonic wave predictions explained the amplitude level variance for non-time-harmonic waves.

We performed a numerical experiment in which the Ricker wavelet was used as non-time-harmonic wave in the FDM simulations (Fig. 9). The results of both the 1.5- and $3-\mathrm{Hz}$ simulations showed no apparent differences in the amplitude level variance between the two wave types in the weak wavefield fluctuation regime $\left(\sigma_{X}^{2}<0.17\right)$. However, in the strong wavefield fluctuation regime, the amplitude level variance was larger for the Ricker wavelet than that for quasi-time-harmonic waves; therefore, amplitude fluctuations may depend on the duration of the source wavelet.

To evaluate the dependence of the duration of source time function on the amplitude level variance, we conducted FDM simulations using different sin waves, which had 1, 2, 3, 6, and 9 s durations (no. 2-6 source 


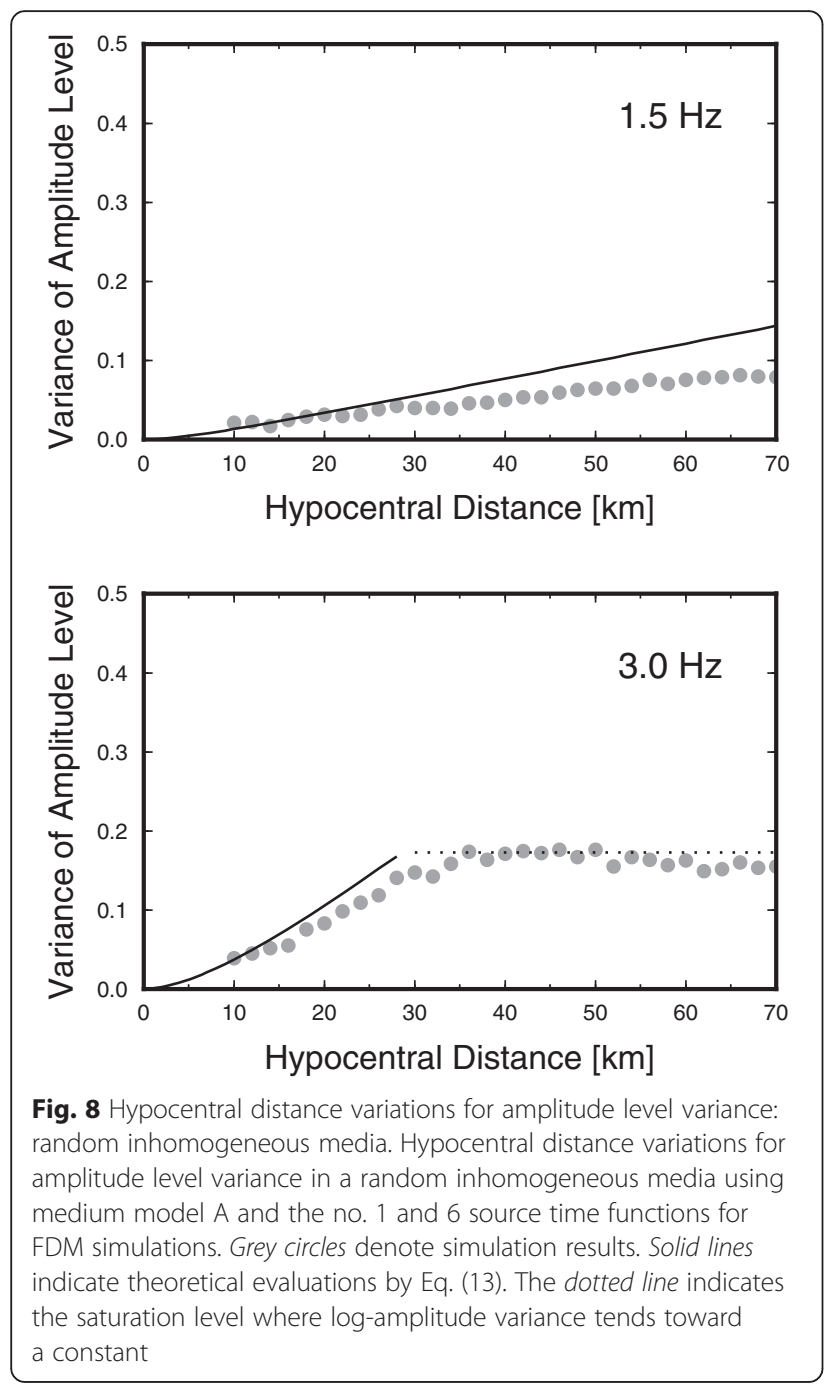

time functions). The amplitude level variance in the weak wavefield fluctuation regime was very similar among the different durations of source time function (Fig. 10a); however, the values in the strong wavefield fluctuation regime increased with the decreasing duration of the source time function. As discussed, the logamplitudes for quasi-time-harmonic waves resembled a normal distribution (Fig. 7), and this characteristic was also observed for non-time-harmonic waves with shorter durations (Fig. 10b); however, the peak of the distribution shifted to smaller log-amplitudes concurrently with the expansion of the left-side tail of the distribution toward the same side. This finding implies that increases in amplitude level variance may be due to insufficient energy supply for the constructive interference of short duration source-radiated wavelets.

Our findings showed that Eq. (13) with an adequate saturation limit may be practically effective for estimating the amplitude level variance of not only timeharmonic waves, but also non-time-harmonic waves,
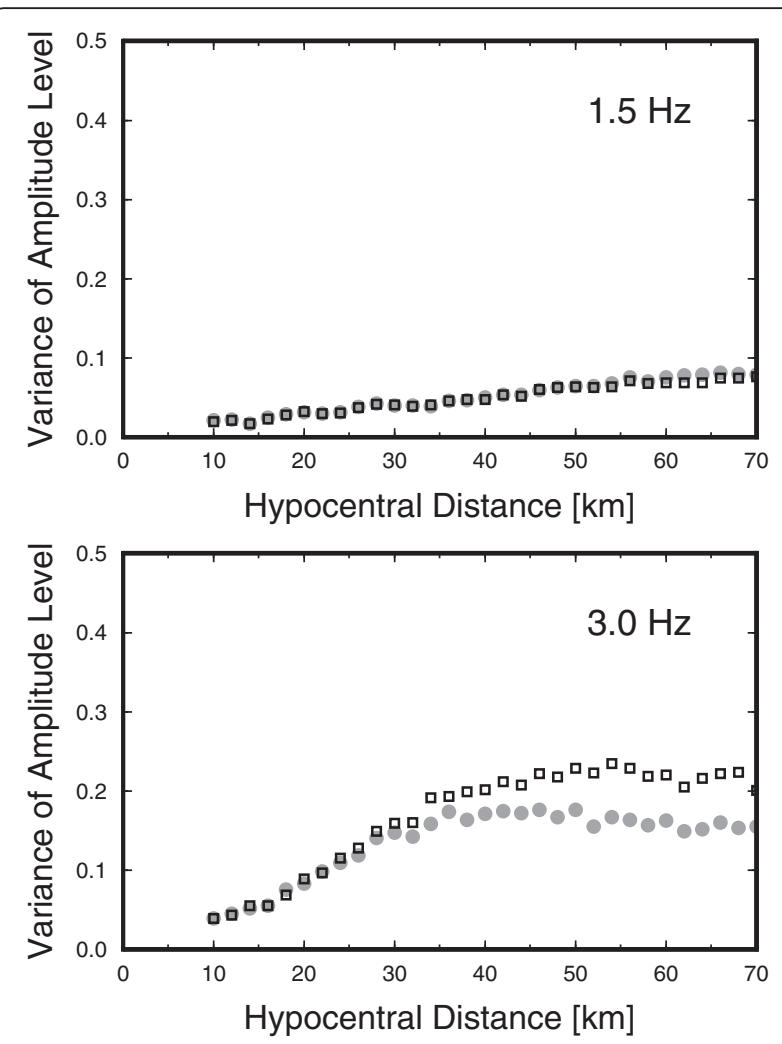

Fig. 9 Hypocentral distance variations of amplitude level variance for different source time functions. Hypocentral distance variations of amplitude level variance for different source time functions using medium model $A$ and the no. 1, 6, 7, and 8 source time functions for FDM simulations. Grey circles show the simulation results for the no. 1 and 6 source time functions (sin wave). Open squares show simulation results for the no. 7 and 8 source time functions (Ricker wavelet)

despite the slight under estimation in the strong wavefield fluctuation regime for non-time-harmonic waves.

\section{Amplitude fluctuations: medium-type dependence}

In addition to numerical analyses using random acoustic media, we conducted additional FDM numerical simulations using elastic random media in order to investigate the dependence of elastic parameter fluctuations on the amplitude level variance.

The hypocentral distance variation of the amplitude level variance obtained for the elastic random media was very similar to that obtained for the acoustic random media, except for small differences in absolute values (Fig. 11b). The ratio of the amplitude level variance of the acoustic random media to that of the elastic random media had a constant value $\sim 0.8$, irrespective of hypocentral distance (Fig. 11b). This indicates that amplitude variation was systematically smaller for the elastic random media than for the acoustic random media. While the physical and quantitative interpretations of this 


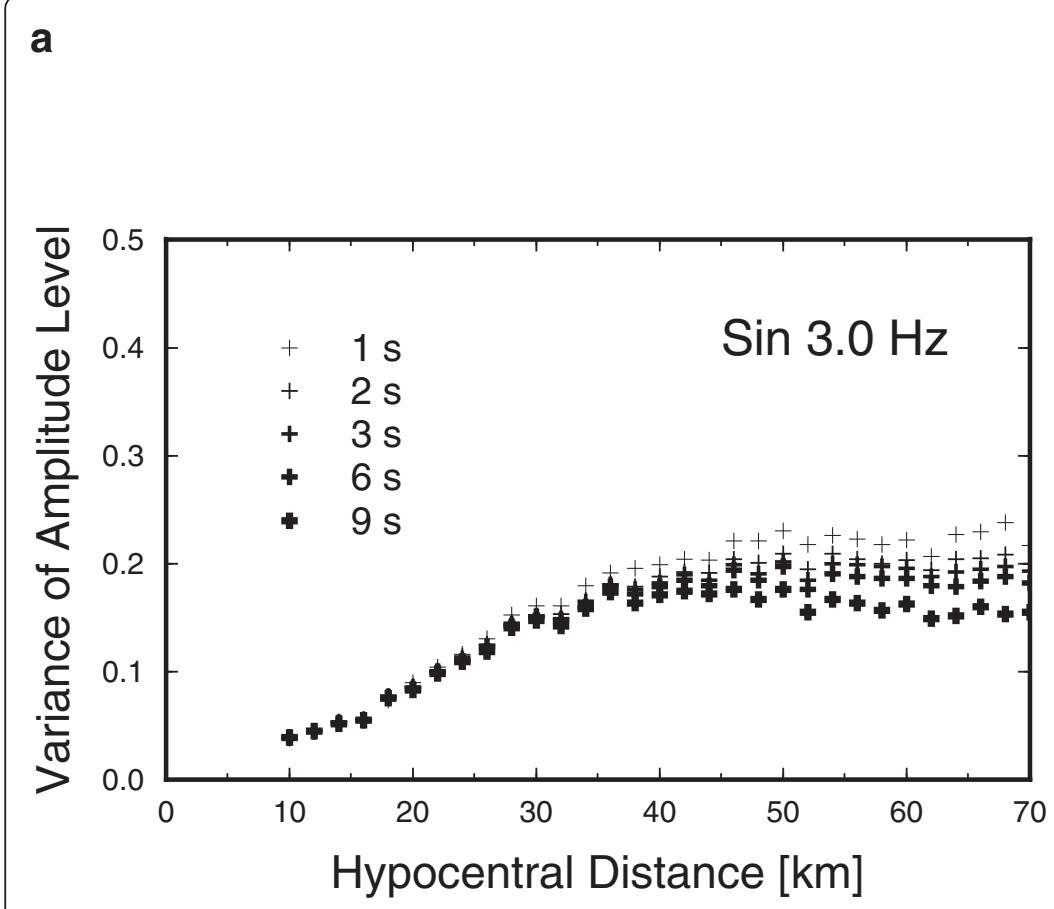

b
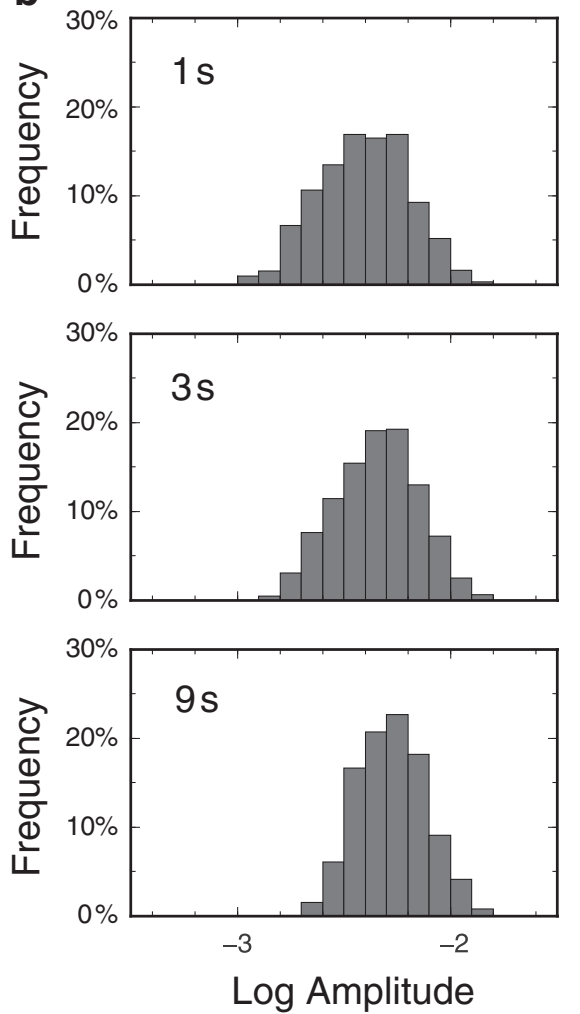

Fig. 10 Amplitude variations for different source time durations. a Hypocentral distance variations in amplitude level variance using medium model $A$ and the no. 2, 3, 4, 5, and 6 source time functions for FDM simulations. The differences in source time duration are shown by the thickness of the symbols. $\mathbf{b}$ Log-amplitude distributions at a distance of $70 \pm 1 \mathrm{~km}$, as obtained from the no. 2, 4, and 6 source time functions. The log-amplitudes are shown using an arbitrary unit

result remain an open question, it may allow us to apply theoretical estimation of Eq. (13) to elastic wave analysis.

We compared the hypocentral distance variations of the amplitude level variance of Ricker wavelets using three random inhomogeneous media (Fig. 12): acoustic random media, elastic random media, and elastic random media without density fluctuations (models A, B, and $C$, respectively). When comparing the acoustic and elastic random media, we found a result similar to that seen for quasi-time-harmonic waves: the amplitude variation was systematically smaller for the elastic random media than for acoustic random media, although this characteristic became obscure at large hypocentral distances $(>50 \mathrm{~km})$. This result was also very similar to that of the comparison between the elastic random media and the elastic random media without density fluctuations. This indicates that the main cause of the amplitude fluctuations in weak random elastic media is not density fluctuations but velocity fluctuations, implying that phase modulation, focusing, and defocusing due to complex ray-bending are crucial for amplitude fluctuation.

\section{Predictions for spherical waves and plane waves}

Hypocentral distance variations in the ratio of the amplitude level variance between spherical and plane waves were investigated using different wavenumbers $(\pi / 4, \pi / 2$, $\pi, 2 \pi$, and $\left.4 \pi \mathrm{km}^{-1}\right)$. The results for plane waves were estimated by setting $\gamma=1$ in Eq. (13). We assumed an exponential-type acoustic random media with $a=1 \mathrm{~km}$ and $\varepsilon=0.03$ (model A). The wavenumber value $\pi \mathrm{km}^{-1}$ roughly corresponded to the $3-\mathrm{Hz}$ frequency in the previous FDM simulations. The ratio was invariably greater than one (Fig. 13), indicating that the amplitude level variance of plane waves was always greater than that of spherical waves. The difference was especially distinct at small hypocentral distances and for high wavenumber waves, which was a reasonable result given that plane waves suffer the effects of wave propagation across a much wider inhomogeneous volume than do spherical waves. These results indicate that for analyzing amplitude variations of seismic waves at small hypocentral distances and in the high-frequency range, a formulation developed for spherical wave propagation is required for data analysis. 


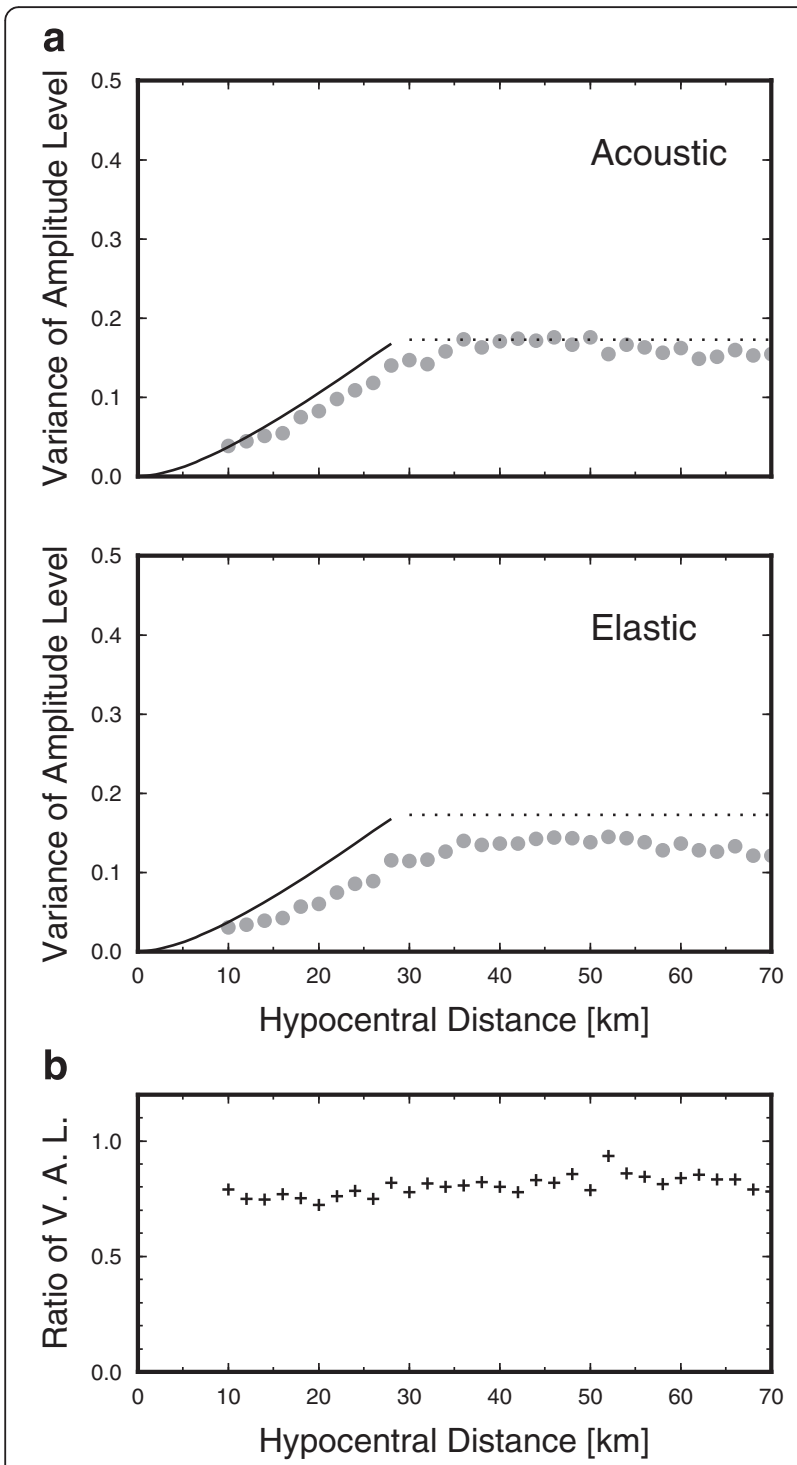

Fig. 11 Hypocentral distance variations for amplitude level variance in different random inhomogeneous media. Hypocentral distance variations for amplitude level variance in different random inhomogeneous media using medium models $A$ and $B$ and the no. 8 source time function for FDM simulations. a Results of FDM simulations for acoustic and elastic media. Grey circles show simulation results. Solid lines indicate theoretical evaluations by Eq. (13). The dotted line indicates the saturation level where log-amplitude variance tends to a constant. b Ratio between amplitude level variance for medium model A (acoustic medium) and medium model B (elastic medium)

\section{Analytical predictions and observational results}

The amplitude level variance for the observed $P$-waves increased with increasing hypocentral distance (Fig. 14a). The rates of increase and absolute values of the amplitude level variance were smaller for $1-2-\mathrm{Hz}$ waves than those for 2-4- $\mathrm{Hz}$ waves. For the lower-frequency waves, the absolute values of the amplitude level variance monotonically increased over the whole hypocentral

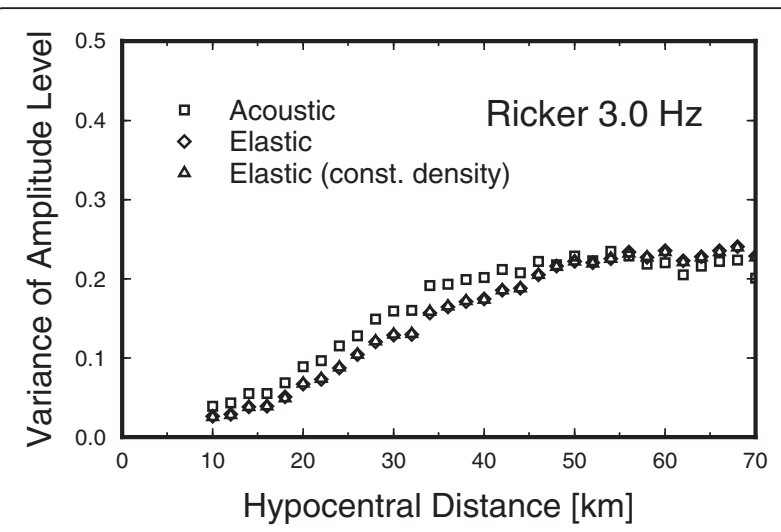

Fig. 12 Hypocentral distance variations for amplitude level variance in different random inhomogeneous media. Hypocentral distance variations for amplitude level variance in different random inhomogeneous media using medium models $A, B$, and $C$ and the no. 8 source time function for FDM simulations. Open squares denote the results for acoustic medium, open diamonds denote the elastic medium, and the open triangles denote the elastic (contrast density) medium

distance range, despite large data scatter. In contrast, for the $2-4-\mathrm{Hz}$ waves, the increases in the absolute values of variance became saturated at a distance of $\sim 30 \mathrm{~km}$. These characteristics were successfully explained by the analytical evaluations of Eq. (13).

Despite the small data volume, the log-amplitude distribution of $P$-waves observed at hypocentral distance of $70 \mathrm{~km}$ appeared to show a normal distribution rather than a uniform distribution (Fig. 14b). This characteristic was consistent with the results obtained by the FDM simulations (Figs. 7 and 10b); furthermore, the wider distribution for high-frequency waves was also consistent with the FDM simulation results.

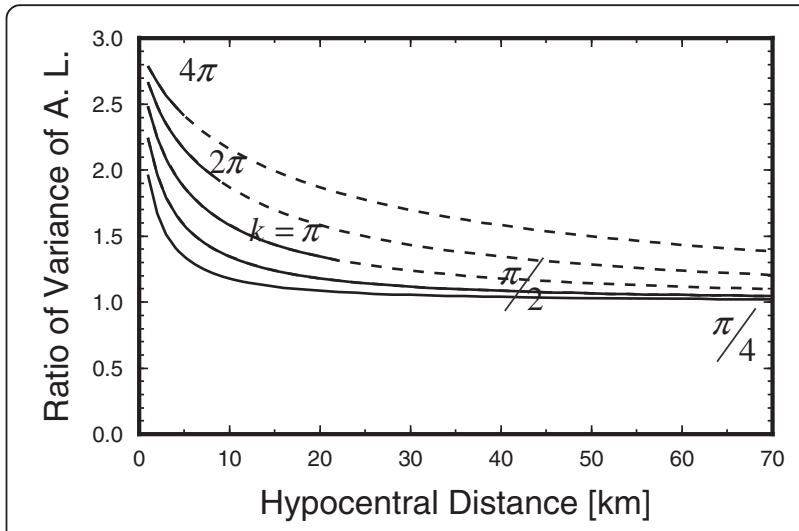

Fig. 13 Differences in amplitude level variance between spherical and plane waves. Ratio of amplitude level variance for spherical waves to that of plane waves for wavenumber values of $\pi / 4, \pi / 2, \pi$, $2 \pi$, and $4 \pi \mathrm{km}^{-1}$. Stochastic parameters $a=1 \mathrm{~km}$ and $\varepsilon=0.03$ are assumed. The dotted line indicates the region in which data are at saturation level 


\section{a}
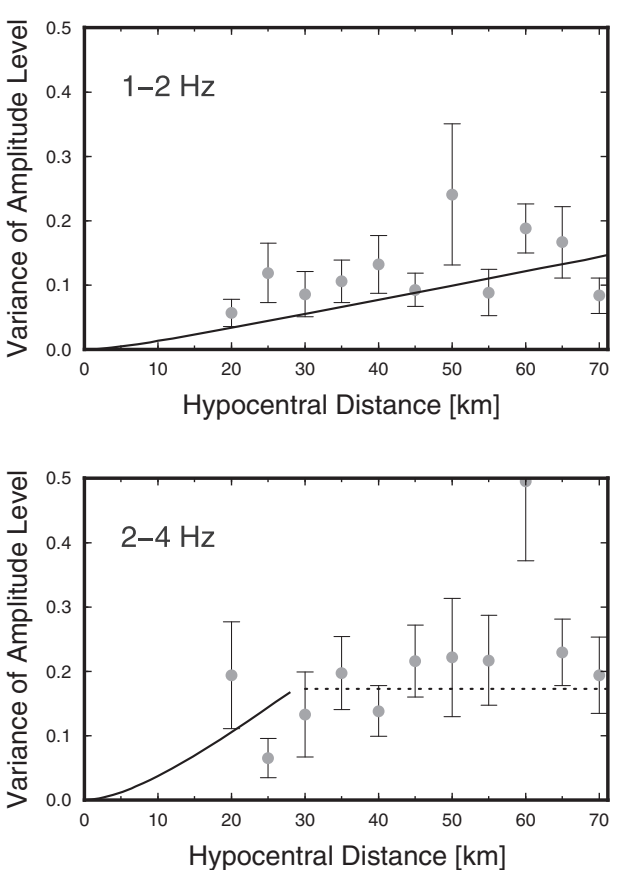

b
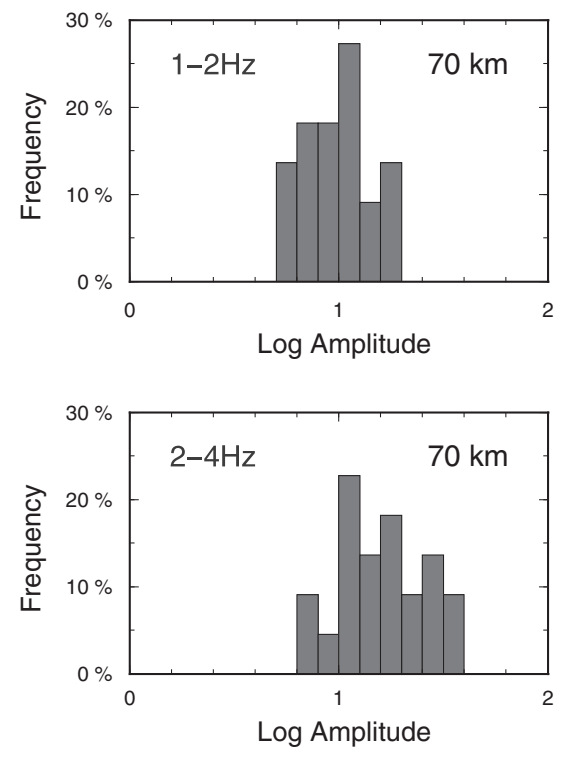

Fig. 14 Observed variations in the $P$-wave amplitude of local earthquakes. a Hypocentral distance variations in the amplitude level variance of observed P-waves. Grey circles show the observation results of local earthquakes (Fig. 2b). Error bars represent $68 \%$ confidence intervals from bootstrap sampling. Solid lines indicate theoretical evaluations by Eq. (13), where medium model A and the no. 1 and 6 source time functions were adopted. The dotted line indicates the saturation level. $\mathbf{b}$ Log-amplitude distributions at a hypocentral distance of $70 \pm 2.5 \mathrm{~km}$, as obtained from seismic observations (Figs. 1 and 2b). The log-amplitudes are shown using an arbitrary unit

\section{Focus of future analyses}

The FDM simulations in this study demonstrated that variations of wave amplitude in random inhomogeneous media depend on not only the inhomogeneity of the medium but also on the duration of the source wavelet. Thus, to use analytical Eq. (13) for the analysis of $P$ waves from local and/or regional earthquakes, it is important to consider the source duration of a target earthquake, which should change by earthquake size or magnitude. In this study, in order to minimize this effect, we selected similar size earthquakes for analysis. We anticipate that predictions by Eq. (13), which deals with quasi-time-harmonic waves, will be more suitable for data from large earthquakes with long source time durations. However, a detailed quantitative analysis of earthquake-size dependence on $P$-wave amplitude variations will be the focus of future work.

In this study, we also restricted our focus to $P$-wave analysis. Thus, applying analytical estimations using Eq. (13) to strong ground motion prediction is not straightforward, in particular because peak ground motions (e.g., peak ground velocity) are mainly excited by $S$ waves (e.g., Atkinson and Mereu 1992). However, we believe that estimations from Eq. (13) are practically useful for the evaluation of $S$-wave amplitude variations in inhomogeneous crust because the Rytov approximation has been successfully applied to the analysis of vector waves (e.g., electromagnetic wave propagation; Ishimaru 1997). From the analysis of strong motion data, Midorikawa and Ohtake (2003) reported that the variance of peak horizontal accelerations and velocities increased with increasing hypocentral distance over a short range $(<50 \mathrm{~km})$, but decreased with increasing earthquake magnitude. These characteristics are consistent with those found for $P$-waves in this analysis, suggesting the applicability of our methodology to strong ground motion prediction in earthquake engineering.

\section{Conclusions}

To better understand the amplitude fluctuations of highfrequency seismic waves, we carried out observational, theoretical, and numerical analyses of waves propagating in random inhomogeneous media.

Our seismic observations of the $P$-wave amplitudes of small to moderately sized crustal earthquakes revealed that the fluctuation of $P$-wave amplitude increased with increasing hypocentral distance, with up to ten-times difference between the largest and the smallest $P$-wave amplitudes. The saturation of this phenomenon occurred at certain hypocentral distances. Furthermore, the observed 
amplitude fluctuations and their rate of increase with the hypocentral distance increased with increasing frequency.

Our theoretical studies presented an analytical equation for evaluating the amplitude fluctuation of timeharmonic waves isotropically radiated by a point source and spherically propagating in acoustic von Kármán-type random media. The equation approximately explained the characteristics of $P$-wave amplitude fluctuations observed from local crustal earthquake as a function of frequency and hypocentral distance. Using this equation, we confirmed the differences in amplitude fluctuation between spherical and plane waves. It was verified that the amplitude fluctuations of plane waves are greater than those of spherical waves when compared at the same travel distance from source.

Our numerical analyses based on FDM simulations enabled us to investigate the characteristics of wave propagation in both acoustic and elastic random media using a variety of source time functions. The results of the simulation showed that the hypocentral distance variations of the amplitude fluctuations obtained for the elastic random media were similar to those obtained for the acoustic random media, except for some differences in absolute values (i.e., the amplitude fluctuation was slightly smaller for the elastic random medium than for the acoustic random medium). We found that the values of the variance of the amplitude level in the weak wavefield fluctuation regime were very similar among the different source time function durations; however, the values in the strong wavefield fluctuation regime increased with decreasing duration. These results indicate that our analytical equation for amplitude fluctuations may be useful in the analysis of substantial seismic data.

\section{Competing interests}

The authors declare that they have no competing interests.

\begin{abstract}
Authors' contributions
KY carried out the theoretical consideration of amplitude fluctuations in an inhomogeneous media and drafted the manuscript. ST conducted the 3D FDM simulations and helped draft the manuscript. MK analyzed the observed seismograms of the Hi-net/F-net. All authors approved the final manuscript.
\end{abstract}

\section{Acknowledgements}

We thank two anonymous reviewers, and the editor, Takuto Maeda, for constructive comments that improved an earlier draft of this manuscript. We acknowledge the National Research Institute for Earth Science and Disaster Prevention, Japan for providing the Hi-net/F-net waveform data and the CMT solutions from the F-net. We also used the unified hypocentral catalog provided by the Japan Meteorological Agency. The FDM simulations of seismic wave propagation were conducted on the computer system of the Earthquake and Volcano Information Center at the Earthquake Research Institute, The University of Tokyo. The authors are particularly indebted to Haruo Sato and Hisashi Nakahara for valuable comments and discussions concerning wave propagation in inhomogeneous media. All figures were drawn using the Generic Mapping Tools software package developed by Wessel and Smith (1998).

\section{Author details}

${ }^{1}$ Department of Material System Science, Graduate School of

Nanobioscience, Yokohama City University, 22-2, Seto, Kanazawa-ku, Yokohama 236-0027, Japan. ${ }^{2}$ National Research Institute for Earth Science and Disaster Prevention, 3-1, Tennodai, Tsukuba, Ibaraki 305-0006, Japan.

Received: 17 October 2015 Accepted: 28 November 2015

Published online: 10 December 2015

\section{References}

Abramowitz M, Stegun IA (1964) Handbook of mathematical functions: with formulas, graphs, and mathematical tables. U. S. Govt. Printing Office, Washington

Aki K, Richards P (2002) Quantitative seismology (2nd edition). University Science Books, Freeman, San Francisco

Anderson JG, Uchiyama Y (2011) A methodology to improve ground-motion prediction equations by including path corrections. Bull Seismol Soc Am 101(4):1822-1846. doi:10.1785/0120090359

Andrews LC, Phillips RL (2005) Laser beam propagation through random media, 2nd edn. SPIE Publications, Washington

Atkinson GM (2006) Single-station sigma. Bull Seismol Soc Am 96(2):446-455. doi:10.1785/0120050137

Atkinson GM, Mereu RF (1992) The shape of ground motion attenuation curves in southeastern Canada. Bull Seismol Soc Am 82(5):2014-2031

Birch AF (1961) The velocity of compressional waves in rocks to 10 kilobars, part 2. J Geophys Res 66:2199-2224. doi:10.1029/JZ065i004p01083

Brillinger DR, Preisler HK (1984) An exploratory analysis of the Joyner-Boore attenuation data. Bull Seismol Soc Am 74(4):1441-1450

Chen YH, Tsai CCP (2002) A new method for estimation of the attenuation relationship with variance components. Bull Seismol Soc Am 92(5):1984-1991. doi:10.1785/0120010205

Douglas J (2003) Earthquake ground motion estimation using strong motion records: a review of equations for the estimation of peak ground acceleration and response spectral ordinates. Earth Sci Rev 61:43-104. doi:10.1016/S0012-8252(02)00112-5

Flatté SM, Wu RS (1988) Small-scale structure in the lithosphere and asthenosphere deduced from arrival time and amplitude fluctuations at NORSAR. J Geophys Res 93:6601-6614. doi:10.1029/JB093iB06p06601

Fukuyama E, Ishida M, Dreger DS, Kawai H (1998) Automated seismic moment tensor determination by using on-line broadband seismic waveforms. Zisin 51:149-156 (in Japanese with English abstract)

Furumura T, Chen L (2004) Large scale parallel simulation and visualization of 3D seismic wavefield using Earth simulator. Comput Model Eng Sci 6:153-168

Hoshiba M (2000) Large fluctuation of wave amplitude produced by small fluctuation of velocity structure. Phys Earth Planetary Int 120(3):201-217. doi:10.1016/S0031-9201(99)00165-X

Ishimaru A (1997) Wave propagation and scattering in random media. IEEE Press and Oxford University Press, ISBN: 078034717X.

Kobayashi M, Takemura S, Yoshimoto K (2015) Frequency and distance changes in the apparent P-wave radiation pattern: effects of seismic wave scattering in the crust inferred from dense seismic observations and numerical simulations. Geophys J Int 202:1895-1907. doi:10.1093/gji/ggv263

Lin PS, Chiou B, Abrahamson N, Walling M, Lee CT, Cheng CT (2011) Repeatable source, site, and path effects on the standard deviation for empirical ground-motion prediction models. Bull Seismol Soc Am 101(5):2281-2295. doi:10.1785/0120090312

Mavroeidis GP, Papageorgiou AS (2003) A mathematical representation of near-fault ground motions. Bull Seismol Soc Am 93(3):1099-1131. doi:10.1785/0120020100

Midorikawa S, Ohtake Y (2003) Empirical analysis of variance of ground motion intensity in attenuation relationships. J Japan Assoc Earthquake Eng 3(1):59-70, http://doi.org/10.5610/jaee.3.59 (in Japanese with English abstract)

Müller TM, Shapiro SA (2003) Amplitude fluctuations due to diffraction and refraction in anisotropic random media: implications for seismic scattering attenuation estimates. Geophys J Int 155(1):139-148. doi:10.1046/j.1365-246X. 2003.02020.x

Nikolaev A V (1975) The seismics of heterogeneous and turbid media (Engl. trans. by Hardin R), Israel program for science translations, Jerusalem

Okada Y, Kasahara K, Hori S, Sekiguchi S, Fujiwara H, Yamamoto A (2004) Recent progress of seismic observation networks in Japan-Hi-net, F-net, K-NET and KiK-net-, Earth Planets Space 56, xv-xxviii 
Ripperger J, Mai PM, Ampuero JP (2008) Variability of near-field ground motion from dynamic earthquake rupture simulations. Bull Seismol Soc Am 98(3): 1207-1228. doi:10.1785/0120070076

Ritter JRR, Shapiro SA, Schechinger B (1998) Scattering parameters of the lithosphere below the Massif Central, France, from teleseismic wavefield records. Geophys J Int 134(1):187-198. doi:10.1046/j.1365-246x.1998.00562.x

Rytov SM, Kravtsov YA, Tatarskii VI (1989) Principles of statistical radiophysics, Vol. 4, Wave propagation through random media. Springer-Verlag, Berlin

Sato H, Fehler MC, Maeda T (2012) Seismic wave propagation and scattering in the heterogeneous earth: the second edition, 1-494. Springer Verlag, Berlin Hidelberg

Shapiro SA, Kneib G (1993) Seismic attenuation by scattering: theory and numerical results. Geophys J Int 114:373-391. doi:10.1111/j.1365-246X.1993. tb03925.x

Si H, Midorikawa S (1999) New attenuation relationships for peak ground acceleration and velocity considering effects of fault type and site condition. J Struct Constr Eng AlJ 523:63-70 (in Japanese with English abstract)

Strasser FO, Abrahamson NA, Bommer JJ (2009) Sigma: issues, insights, and challenges. Seismol Res Lett 80:40-56. doi:10.1785/gssrl.80.1.40

Takemura S, Furumura T, Maeda T (2015) Scattering of high-frequency seismic waves caused by irregular surface topography and small-scale velocity inhomogeneity. Geophys J Int 201:459-474. doi:10.1093/gji/ggv038

Uscinski BJ (1977) Elements of wave propagation in random media. McGraw-Hill, New York

Wessel P, Smith WHF (1998) New improved version of generic mapping tools released. EOS Trans Amer Geophys Union 79(47):579. doi:10.1029/98EO00426

Yoshimoto K, Takemura S (2014) A study on the predominant period of longperiod ground motions in the Kanto Basin, Japan. Earth Planets Space 66:100. doi:10.1186/1880-5981-66-100

Yoshimoto K, Sato H, Ohtake M (1993) Frequency-dependent attenuation of P and $\mathrm{S}$ waves in the Kanto area, Japan, based on the coda-normalization method. Geophys J Int 114:165-174. doi:10.1111/j.1365-246X.1993.tb01476.X

\section{Submit your manuscript to a SpringerOpen ${ }^{\odot}$ journal and benefit from:}

- Convenient online submission

- Rigorous peer review

- Immediate publication on acceptance

- Open access: articles freely available online

- High visibility within the field

- Retaining the copyright to your article

Submit your next manuscript at $\gg$ springeropen.com 